\title{
A NOTE ON 'IMPROVED FRÉCHET BOUNDS AND MODEL-FREE PRICING OF MULTI-ASSET OPTIONS' BY TANKOV (2011)
}

\author{
CAROLE BERNARD *** AND \\ XIAO JIANG, ${ }^{* * * *}$ University of Waterloo \\ STEVEN VANDUFFEL, ${ }^{* * * *}$ Vrije Universiteit Brussel
}

\begin{abstract}
Tankov (2011) improved the Fréchet bounds for a bivariate copula when its values on a compact subset of $[0,1]^{2}$ are given. He showed that the best possible bounds are quasicopulas and gave a sufficient condition for these bounds to be copulas. In this note we give weaker sufficient conditions to ensure that the bounds are copulas. We also show how this can be useful in portfolio selection. It turns out that finding a copula as a lower bound plays a key role in determining optimal investment strategies explicitly for investors with some type of state-dependent constraints.
\end{abstract}

Keywords: Copula; Fréchet-Hoeffding bound; quasi-copula; optimal portfolio selection 2010 Mathematics Subject Classification: Primary 60E15; 62P05; 91G10

\section{Introduction}

Unless otherwise stated, in this note we adopt all the notation and conventions of Tankov (2011). Hence, let $Q:[0,1]^{2} \rightarrow[0,1]$ be a quasi-copula, i.e. $Q$ satisfies the following properties.

(i) Boundary conditions: $Q(0, u)=Q(u, 0)=0$ and $Q(1, u)=Q(u, 1)=u$ for all $u \in[0,1]$.

(ii) $Q$ is increasing in each argument (strictly speaking, $Q$ is nondecreasing in each argument).

(iii) Lipschitz property: $\left|Q\left(u_{2}, v_{2}\right)-Q\left(u_{1}, v_{1}\right)\right| \leq\left|u_{2}-u_{1}\right|+\left|v_{2}-v_{1}\right|$ for all $\left(u_{1}, v_{1}\right.$, $\left.u_{2}, v_{2}\right) \in[0,1]$.

Note that if, in addition, $Q$ is also 2-increasing then it is a copula. We recall that $Q$ is 2-increasing if $V_{Q}(R)=Q\left(u_{2}, v_{2}\right)+Q\left(u_{1}, v_{1}\right)-Q\left(u_{1}, v_{2}\right)-Q\left(u_{2}, v_{1}\right) \geq 0$ for every rectangle $R=\left[u_{1}, u_{2}\right] \times\left[v_{1}, v_{2}\right] \subseteq[0,1]^{2}$. Furthermore, let $S$ denote a compact subset of the unit square $[0,1]^{2}$ and let $Q_{S}$ be the set of all quasi-copulas $Q^{\prime}$ such that $Q^{\prime}(a, b)=Q(a, b)$ for all $(a, b) \in S$. Tankov (2011) considered the problem of finding best possible bounds for

Received 26 July 2011; revision received 12 February 2012.

* Postal address: Department of Statistics and Actuarial Science, University of Waterloo, 200 University Avenue West, Waterloo ON, N2L 3G1, Canada.

** Email address: c3bernar@uwaterloo.ca

*** Email address: xiaopz0@gmail.com

**** Postal address: Faculty of Economics, Vrije Universiteit Brussel, Pleinlaan 2, 1050 Elsene, Belgium.

Email address: steven.vanduffel@vub.ac.be 
the set $Q_{S}$. Of course, when $S$ is the empty set, every quasi-copula $Q^{\prime}$ satisfies the FréchetHoeffding bounds, i.e.

$$
W(u, v):=\max \{0, u+v-1\} \leq Q^{\prime}(u, v) \leq \min \{u, v\}:=M(u, v) .
$$

In the general case this problem appears more challenging; see Nelsen (2006) for best possible bounds when $S$ is a singleton. Tankov (2011) defined

$$
\begin{aligned}
& A^{S, Q}(u, v):=\min \left\{u, v, \min _{(a, b) \in S}\left\{Q(a, b)+(u-a)^{+}+(v-b)^{+}\right\}\right\}, \\
& B^{S, Q}(u, v):=\max \left\{0, u+v-1, \max _{(a, b) \in S}\left\{Q(a, b)-(a-u)^{+}-(b-v)^{+}\right\}\right\},
\end{aligned}
$$

where $(u, v) \in[0,1]^{2}$, and showed in his Theorem 1 that $A^{S, Q}$ and $B S, Q$ are respectively best possible upper and lower bounds, and thus improve the Fréchet-Hoeffding upper and lower bounds, respectively. Note that improvement results for the Fréchet-Hoeffding bounds when there are additional (inequality) constraints have already been given in Rachev and Rüschendorf (1994) and discussed extensively in Section 7.3 of Rachev and Rüschendorf (1998). Tankov (2011) also demonstrated that a sufficient condition for $A^{S, Q}$ or $B$ S, $Q$ to be a copula is to suppose that $S$ is nonincreasing or, respectively, nondecreasing. In this note, we extend this result by showing that, when $Q$ is a copula, $A^{S, Q}$ or $B$ ' $Q$ is a copula when $S$ is a compact set satisfying some additional conditions, namely a 'nonincreasingness' or, respectively, 'nondecreasingness' and a 'connectivity' property. For instance, when $S$ is a rectangle, then both $A^{S, Q}$ and $B S, Q$ are copulas.

Theorem 1 of Tankov (2011) and our additions to it are of interest in finance. Tankov already demonstrated how his results are instrumental in finding model-free bounds for the prices of some two-asset derivatives. He showed how information embedded in the financial market (such as the price of another two-asset option) translates into extra information about dependence, and thus leads to sharper traditional bounds for prices, which are based on FréchetHoeffding bounds on copulas (where information on dependence is ignored). In this note we show that the study of optimal investment strategies is intimately connected to finding bounds on the dependence between the final wealth and the so-called stochastic discount factor (pricing kernel or state-price process) at maturity. In particular, knowing that $B S, Q$ is a copula is useful to determine investment strategies that are optimal for investors with state-dependent constraints, i.e. when they do not only care about the distribution of final wealth but also about the states where cash flows are received. More details are given in Section 3. Both mentioned applications make clear that it is of interest to know more situations for which the bounds appearing in Tankov (2011) are copulas (see Theorems 2.1, 2.2, and 2.3 in this note).

\section{Extensions of Theorem 1 of Tankov (2011)}

In this section we extend Theorem 1 of Tankov (2011). To this end, we need the following lemma.

Lemma 2.1. Assume that $f:[0,1]^{2} \mapsto \mathbb{R}$ is 2-increasing, nondecreasing in each argument, and satisfies the Lipschitz property. Define the function $g:[0,1]^{2} \mapsto \mathbb{R}$ as

$$
g=\max \{f, W\},
$$

where $W(u, w)=\max \{u+v-1,0\}$ is the anti-monotonic copula. Then $g$ is also 2-increasing, nondecreasing in each argument, and satisfies the Lipschitz property. 
Proof. Note that $W$ satisfies the Lipschitz property. Hence, $g$, as the maximum of two functions with the Lipschitz property, also satisfies the Lipschitz property (as shown in part (i) of the proof of Theorem 1 of Tankov (2011)). It is obvious that $g$ is also nondecreasing in each argument. In order to prove that $g$ is 2-increasing, let us consider any rectangle $R=\left[u_{1}, u_{2}\right] \times\left[v_{1}, v_{2}\right]$ in the unit square (we conventionally assume that $u_{1}<u_{2}$ and $\left.v_{1}<v_{2}\right)$. We identify the following three cases.

Case 1: assume that either $\max \left\{f\left(u_{2}, v_{1}\right), u_{2}+v_{1}-1\right\} \leq 0$ or $\max \left\{f\left(u_{1}, v_{2}\right), u_{1}+v_{2}-1\right\} \leq$ 0 . Since both functions are nondecreasing in each argument, we find that

$$
\max \left\{f\left(u_{1}, v_{1}\right), u_{1}+v_{1}-1\right\} \leq 0 .
$$

Without loss of generality, we can take $\max \left\{f\left(u_{2}, v_{1}\right), u_{2}+v_{1}-1\right\} \leq 0$ (the other case is similar). Then the $g$-volume of the rectangle $R$ is given by

$$
\begin{aligned}
V_{g}(R) & =g\left(u_{2}, v_{2}\right)-g\left(u_{2}, v_{1}\right)-g\left(u_{1}, v_{2}\right)+g\left(u_{1}, v_{1}\right) \\
& =g\left(u_{2}, v_{2}\right)-g\left(u_{1}, v_{2}\right) \\
& =\max \left\{f\left(u_{2}, v_{2}\right), W\left(u_{2}, v_{2}\right)\right\}-\max \left\{f\left(u_{1}, v_{2}\right), W\left(u_{1}, v_{2}\right)\right\} \\
& \geq 0,
\end{aligned}
$$

where the last inequality follows from $f\left(u_{2}, v_{2}\right) \geq f\left(u_{1}, v_{2}\right)$ and $W\left(u_{2}, v_{2}\right) \geq W\left(u_{1}, v_{2}\right)$.

For cases 2 and 3, we can now assume that both $\max \left\{f\left(u_{2}, v_{1}\right), u_{2}+v_{1}-1\right\}>0$ and $\max \left\{f\left(u_{1}, v_{2}\right), u_{1}+v_{2}-1\right\}>0$.

Case 2: assume that $f\left(u_{2}, v_{1}\right) \geq u_{2}+v_{1}-1$ and $f\left(u_{1}, v_{2}\right) \geq u_{1}+v_{2}-1$. This implies that $g\left(u_{2}, v_{1}\right)=f\left(u_{2}, v_{1}\right)$ and $g\left(u_{1}, v_{2}\right)=f\left(u_{1}, v_{2}\right)$. Hence, the $g$-volume of the rectangle $R$ satisfies

$$
\begin{aligned}
V_{g}(R) & =g\left(u_{2}, v_{2}\right)-g\left(u_{2}, v_{1}\right)-g\left(u_{1}, v_{2}\right)+g\left(u_{1}, v_{1}\right) \\
& \geq f\left(u_{2}, v_{2}\right)-f\left(u_{2}, v_{1}\right)-f\left(u_{1}, v_{2}\right)+f\left(u_{1}, v_{1}\right) \\
& \geq 0,
\end{aligned}
$$

where the last inequality follows from the 2-increasing property for $f$.

Case 3: assume that $f\left(u_{2}, v_{1}\right)<u_{2}+v_{1}-1$ or $f\left(u_{1}, v_{2}\right)<u_{1}+v_{2}-1$. Without loss of generality, we take $f\left(u_{2}, v_{1}\right)<u_{2}+v_{1}-1$ (the other case is similar). Since $\max \left\{f\left(u_{2}, v_{1}\right), u_{2}+v_{1}-1\right\}>0$, it follows that $u_{2}+v_{1}-1>0$ and, thus, also $u_{2}+v_{2}-1>0$. Furthermore, the Lipschitz property for $f$ then also implies that $f\left(u_{2}, v_{2}\right)<u_{2}+v_{2}-1$. Therefore,

$$
\begin{aligned}
V_{g}(R) & =g\left(u_{2}, v_{2}\right)-g\left(u_{2}, v_{1}\right)-g\left(u_{1}, v_{2}\right)+g\left(u_{1}, v_{1}\right) \\
& =\left(u_{2}+v_{2}-1\right)-\left(u_{2}+v_{1}-1\right)-g\left(u_{1}, v_{2}\right)+g\left(u_{1}, v_{1}\right) \\
& =\left(v_{2}-v_{1}\right)-\left(g\left(u_{1}, v_{2}\right)-g\left(u_{1}, v_{1}\right)\right) \\
& \geq 0,
\end{aligned}
$$

where the last inequality follows from the Lipschitz property for $g$.

Let us denote by $S_{1}$ the set obtained by the first variable projection of the compact set $S$, namely, $u \in S_{1}$ if and only if there exists $v \in(0,1)$ such that $(u, v) \in S$. Similarly, we define $S_{2}$ as the second variable projection. Define the two functions

$$
\begin{aligned}
\gamma_{1}: S_{1} & \rightarrow S_{2}, \\
u & \mapsto \min \{v \mid(u, v) \in S\}
\end{aligned}
$$


and

$$
\begin{aligned}
\gamma_{2}: S_{1} & \rightarrow S_{2}, \\
u & \mapsto \max \{v \mid(u, v) \in S\} .
\end{aligned}
$$

The compactness of $S$ guarantees the existence of the above maxima and minima. The points $\left(u, \gamma_{1}(u)\right)_{u \in S_{1}}$ are the 'lower' boundary points of $S$. Similarly, $\left(u, \gamma_{2}(u)\right)_{u \in S_{1}}$ are the 'upper' boundary points. We are now ready to prove the following result.

Theorem 2.1. Let $Q$ be a copula, and let $S \subseteq[0,1]^{2}$ be a compact set with both $\gamma_{1}$ and $\gamma_{2}$ nondecreasing functions that satisfies the following property:

$$
\left(u, \frac{v_{0}+v_{1}}{2}\right) \in S \quad \text { for all }\left(u, v_{0}\right),\left(u, v_{1}\right) \in S \text {. }
$$

Then $B^{S, Q}$ is a copula.

Proof. Tankov (2011) has already shown that $B^{S, Q}$ is a quasi-copula. We thus need to only show that $B^{S, Q}$ is 2 -increasing. Let us write $B^{S, Q}$ as $\max \left\{f^{S, Q}, W\right\}$, where $f^{S, Q}$ is the function

$$
f^{S, Q}(u, v):=\max _{(a, b) \in S}\left\{Q(a, b)-(a-u)^{+}-(b-v)^{+}\right\} .
$$

Tankov (2011) proved that $f^{S, Q}$ satisfies the Lipschitz condition. Since $f^{S, Q}$ is also nondecreasing in each argument, it remains to prove that it is also 2-increasing. Then, Lemma 2.1 implies that $B^{S, Q}$ is a 2-increasing quasi-copula and, therefore, a copula. Let us consider any rectangular area $R=\left[u_{1}, u_{2}\right] \times\left[v_{1}, v_{2}\right]$. We want to prove that $V_{f, Q}\left(\left[u_{1}, u_{2}\right] \times\left[v_{1}, v_{2}\right]\right) \geq 0$.

By the compactness of $S$, there exist $\left(u_{1}^{*}, v_{2}^{*}\right) \in S$ and $\left(u_{2}^{*}, v_{1}^{*}\right) \in S$ such that

$$
\begin{aligned}
f^{S, Q}\left(u_{1}, v_{2}\right) & =\max _{(a, b) \in S}\left\{Q(a, b)-\left(a-u_{1}\right)^{+}-\left(b-v_{2}\right)^{+}\right\} \\
& =Q\left(u_{1}^{*}, v_{2}^{*}\right)-\left(u_{1}^{*}-u_{1}\right)^{+}-\left(v_{2}^{*}-v_{2}\right)^{+}
\end{aligned}
$$

and

$$
\begin{aligned}
f^{S, Q}\left(u_{2}, v_{1}\right) & =\max _{(a, b) \in S}\left\{Q(a, b)-\left(a-u_{2}\right)^{+}-\left(b-v_{1}\right)^{+}\right\} \\
& =Q\left(u_{2}^{*}, v_{1}^{*}\right)-\left(u_{2}^{*}-u_{2}\right)^{+}-\left(v_{1}^{*}-v_{1}\right)^{+} .
\end{aligned}
$$

Case 1: assume that $\left(u_{1}^{*}, v_{2}^{*}\right)$ and $\left(u_{2}^{*}, v_{1}^{*}\right)$ form a nondecreasing set. Observe that

$$
\begin{aligned}
f^{S, Q}\left(u_{2}, v_{2}\right) & =\max _{(a, b) \in S}\left\{Q(a, b)-\left(a-u_{2}\right)^{+}-\left(b-v_{2}\right)^{+}\right\} \\
& \geq Q\left(u_{1}^{*}, v_{2}^{*}\right)-\left(u_{1}^{*}-u_{2}\right)^{+}-\left(v_{2}^{*}-v_{2}\right)^{+}
\end{aligned}
$$

and

$$
\begin{aligned}
f^{S, Q}\left(u_{1}, v_{1}\right) & =\max _{(a, b) \in S}\left\{Q(a, b)-\left(a-u_{1}\right)^{+}-\left(b-v_{1}\right)^{+}\right\} \\
& \geq Q\left(u_{2}^{*}, v_{1}^{*}\right)-\left(u_{2}^{*}-u_{1}\right)^{+}-\left(v_{1}^{*}-v_{1}\right)^{+} .
\end{aligned}
$$

Then we bound the volume of the rectangle $\left[u_{1}, u_{2}\right] \times\left[v_{1}, v_{2}\right]$ from below as follows:

$$
\begin{aligned}
& f^{S, Q}\left(u_{2}, v_{2}\right)-f^{S, Q}\left(u_{1}, v_{2}\right)-f^{S, Q}\left(u_{2}, v_{1}\right)+f^{S, Q}\left(u_{1}, v_{1}\right) \\
& \geq {\left[Q\left(u_{1}^{*}, v_{2}^{*}\right)-\left(u_{1}^{*}-u_{2}\right)^{+}-\left(v_{2}^{*}-v_{2}\right)^{+}\right]-\left[Q\left(u_{1}^{*}, v_{2}^{*}\right)-\left(u_{1}^{*}-u_{1}\right)^{+}-\left(v_{2}^{*}-v_{2}\right)^{+}\right] } \\
&-\left[Q\left(u_{2}^{*}, v_{1}^{*}\right)-\left(u_{2}^{*}-u_{2}\right)^{+}-\left(v_{1}^{*}-v_{1}\right)^{+}\right] \\
&+\left[Q\left(u_{2}^{*}, v_{1}^{*}\right)-\left(u_{2}^{*}-u_{1}\right)^{+}-\left(v_{1}^{*}-v_{1}\right)^{+}\right]
\end{aligned}
$$




$$
\begin{aligned}
= & Q\left(u_{1}^{*}, v_{2}^{*}\right)-Q\left(u_{1}^{*}, v_{2}^{*}\right)-Q\left(u_{2}^{*}, v_{1}^{*}\right)+Q\left(u_{2}^{*}, v_{1}^{*}\right)+\left[\left(u_{1}^{*}-u_{1}\right)^{+}-\left(u_{1}^{*}-u_{2}\right)^{+}\right] \\
& -\left[\left(u_{2}^{*}-u_{1}\right)^{+}-\left(u_{2}^{*}-u_{2}\right)^{+}\right] \\
= & {\left[\left(u_{1}^{*}-u_{1}\right)^{+}-\left(u_{1}^{*}-u_{2}\right)^{+}\right]-\left[\left(u_{2}^{*}-u_{1}\right)^{+}-\left(u_{2}^{*}-u_{2}\right)^{+}\right] . }
\end{aligned}
$$

Hence, if $u_{1}^{*} \geq u_{2}^{*}$ in (2.4) then $V_{f} s, Q\left(\left[u_{1}, u_{2}\right] \times\left[v_{1}, v_{2}\right]\right) \geq 0$ holds. When $u_{1}^{*}<u_{2}^{*}$, we proceed similarly. Indeed, it also holds that

$$
\begin{aligned}
& f^{S, Q}\left(u_{2}, v_{2}\right) \geq Q\left(u_{2}^{*}, v_{1}^{*}\right)-\left(u_{2}^{*}-u_{2}\right)^{+}-\left(v_{1}^{*}-v_{2}\right)^{+}, \\
& f^{S, Q}\left(u_{1}, v_{1}\right) \geq Q\left(u_{1}^{*}, v_{2}^{*}\right)-\left(u_{1}^{*}-u_{1}\right)^{+}-\left(v_{2}^{*}-v_{1}\right)^{+},
\end{aligned}
$$

and, therefore, we obtain

$$
\begin{aligned}
f^{S, Q} & \left(u_{2}, v_{2}\right)-f^{S, Q}\left(u_{1}, v_{2}\right)-f^{S, Q}\left(u_{2}, v_{1}\right)+f^{S, Q}\left(u_{1}, v_{1}\right) \\
& \geq\left[\left(v_{2}^{*}-v_{2}\right)^{+}-\left(v_{2}^{*}-v_{1}\right)^{+}\right]-\left[\left(v_{1}^{*}-v_{2}\right)^{+}-\left(v_{1}^{*}-v_{1}\right)^{+}\right] \\
& \geq 0
\end{aligned}
$$

where the last inequality in (2.5) follows from the fact that $v_{2}^{*} \leq v_{1}^{*}$, since $\left(u_{1}^{*}, v_{2}^{*}\right)$ and $\left(u_{2}^{*}, v_{1}^{*}\right)$ form a nondecreasing set.

Case 2: assume that $\left(u_{1}^{*}, v_{2}^{*}\right)$ and $\left(u_{2}^{*}, v_{1}^{*}\right)$ form a nonincreasing set. When $u_{1}^{*} \leq u_{2}^{*}$, then $v_{2}^{*} \geq v_{1}^{*}$. By the compactness of $S$, property (2.3) implies that, for each $u \in S_{1}, S$ contains the vertical segment connecting $\left(u, \gamma_{1}(u)\right)$ and $\left(u, \gamma_{2}(u)\right)$. Thus, $\gamma_{1}\left(u_{1}^{*}\right) \leq v_{2}^{*} \leq \gamma_{2}\left(u_{1}^{*}\right)$ and $\gamma_{1}\left(u_{2}^{*}\right) \leq v_{1}^{*} \leq \gamma_{2}\left(u_{2}^{*}\right)$. Moreover, by the nondecreasing property of $\gamma_{1}$ and $\gamma_{2}$, we have $\gamma_{1}\left(u_{1}^{*}\right) \leq \gamma_{1}\left(u_{2}^{*}\right)$ and $\gamma_{2}\left(u_{1}^{*}\right) \leq \gamma_{2}\left(u_{2}^{*}\right)$. Therefore, $\gamma_{1}\left(u_{1}^{*}\right) \leq v_{1}^{*} \leq \gamma_{2}\left(u_{1}^{*}\right)$ and $\gamma_{1}\left(u_{2}^{*}\right) \leq v_{2}^{*} \leq$ $\gamma_{2}\left(u_{2}^{*}\right)$. Hence, $\left(u_{1}^{*}, v_{1}^{*}\right) \in S$ and $\left(u_{2}^{*}, v_{2}^{*}\right) \in S$. Similarly, we can prove that, when $u_{2}^{*}<u_{1}^{*}$, $\left(u_{1}^{*}, v_{1}^{*}\right) \in S$ and $\left(u_{2}^{*}, v_{2}^{*}\right) \in S$.

We obtain, for $\left(u_{1}, v_{1}\right)$ and $\left(u_{2}, v_{2}\right)$,

$$
f^{S, Q}\left(u_{1}, v_{1}\right) \geq Q\left(u_{1}^{*}, v_{1}^{*}\right)-\left(u_{1}^{*}-u_{1}\right)^{+}-\left(v_{1}^{*}-v_{1}\right)^{+}
$$

and

$$
f^{S, Q}\left(u_{2}, v_{2}\right) \geq Q\left(u_{2}^{*}, v_{2}^{*}\right)-\left(u_{2}^{*}-u_{2}\right)^{+}-\left(v_{2}^{*}-v_{2}\right)^{+} .
$$

We can then conclude that the volume of the rectangle $\left[u_{1}, u_{2}\right] \times\left[v_{1}, v_{2}\right]$ is nonnegative because

$$
\begin{aligned}
& f^{S, Q}\left(u_{2}, v_{2}\right)-f^{S, Q}\left(u_{1}, v_{2}\right)-f^{S, Q}\left(u_{2}, v_{1}\right)+f^{S, Q}\left(u_{1}, v_{1}\right) \\
& \geq {\left[Q\left(u_{2}^{*}, v_{2}^{*}\right)-\left(u_{2}^{*}-u_{2}\right)^{+}-\left(v_{2}^{*}-v_{2}\right)^{+}\right]-\left[Q\left(u_{1}^{*}, v_{2}^{*}\right)-\left(u_{1}^{*}-u_{1}\right)^{+}-\left(v_{2}^{*}-v_{2}\right)^{+}\right] } \\
& \quad-\left[Q\left(u_{2}^{*}, v_{1}^{*}\right)-\left(u_{2}^{*}-u_{2}\right)^{+}-\left(v_{1}^{*}-v_{1}\right)^{+}\right] \\
& \quad+\left[Q\left(u_{1}^{*}, v_{1}^{*}\right)-\left(u_{1}^{*}-u_{1}\right)^{+}-\left(v_{1}^{*}-v_{1}\right)^{+}\right] \\
&= Q\left(u_{2}^{*}, v_{2}^{*}\right)-Q\left(u_{1}^{*}, v_{2}^{*}\right)-Q\left(u_{2}^{*}, v_{1}^{*}\right)+Q\left(u_{1}^{*}, v_{1}^{*}\right) \\
& \geq 0
\end{aligned}
$$

where the last inequality follows the fact that $Q$ is 2-increasing, and $\left(u_{1}^{*}, v_{2}^{*}\right)$ and $\left(u_{2}^{*}, v_{1}^{*}\right)$ form a nonincreasing set. We have thus proved that $f^{S, Q}$ is 2 -increasing. Lemma 2.1 implies that $B^{S, Q}$ is a copula. 
The following two functions define the 'left' and 'right' boundary points of $S$ :

$$
\begin{aligned}
\gamma_{3}: S_{2} & \rightarrow S_{1}, \\
v & \mapsto \min \{u \mid(u, v) \in S\}
\end{aligned}
$$

and

$$
\begin{aligned}
\gamma_{4}: S_{2} & \rightarrow S_{1}, \\
v & \mapsto \max \{u \mid(u, v) \in S\} .
\end{aligned}
$$

The following result is dual to Theorem 2.1: its proof is obtained by symmetry.

Theorem 2.2. Let $Q$ be a copula, and let $S \subseteq[0,1]^{2}$ be a compact set with both $\gamma_{3}$ and $\gamma_{4}$ nondecreasing functions that satisfies the following property:

$$
\left(\frac{u_{0}+u_{1}}{2}, v\right) \in S \quad \text { for all }\left(u_{0}, v\right),\left(u_{1}, v\right) \in S .
$$

Then $B^{S, Q}$ is a copula.

Remark 2.1. The conditions in Theorems 2.1 and 2.2 cannot be readily relaxed. Indeed, consider $S=\{A, B, C, D\}$, where $A=\left(\frac{1}{3}, 0\right), B=\left(\frac{1}{3}, \frac{2}{3}\right), C=\left(\frac{2}{3}, \frac{1}{3}\right)$, and $D=\left(\frac{2}{3}, 1\right)$, and let $Q=\min \{u, v\}$. Note that property (2.3) is not satisfied and also that $\gamma_{3}$ (as well as $\gamma_{4}$ ) is not nondecreasing, so neither Theorem 2.1 nor Theorem 2.2 can be invoked to show that $B S, Q$ is a copula. We observe that $B, Q$ is indeed not a copula because

$$
B^{S, Q}\left(\frac{2}{3}, \frac{2}{3}\right)-B^{S, Q}\left(\frac{2}{3}, \frac{1}{3}\right)-B^{S, Q}\left(\frac{1}{3}, \frac{2}{3}\right)+B^{S, Q}\left(\frac{1}{3}, \frac{1}{3}\right)=\frac{1}{3}-\frac{1}{3}-\frac{1}{3}+0=-\frac{1}{3}<0 .
$$

Remark 2.2. At first glance, part (ii) of Theorem 1 of Tankov (2011) does not appear to always follow from Theorem 2.1 or Theorem 2.2. For example, let us consider the compact set $S=\{A, B, C\}$, where $A=\left(x_{1}, y_{1}\right), B=\left(x_{2}, y_{1}\right)$, and $C=\left(x_{2}, y_{2}\right)$, with $x_{1}<x_{2}$ and $y_{1}<y_{2}$. Then neither property (2.3) nor property (2.6) is satisfied. Nevertheless, $S$ is a nondecreasing compact set for which part (ii) of Theorem 1 of Tankov (2011) can be applied, implying that $B, Q$ is a copula. However, we can also use our results combined with a limiting argument to obtain the same result. Consider $S_{n}=\left\{A, B_{n}, C\right\}$, where $A=\left(x_{1}, y_{1}\right)$, $B_{n}=\left(x_{2}-\left(x_{2}-x_{1}\right) / n, y_{1}\right)$, and $C=\left(x_{2}, y_{2}\right)$. Then, using Theorem 2.1, for all positive $n \in \mathbb{N}, B^{S_{n}, Q}$ is a copula. Moreover,

$$
\begin{aligned}
B^{S_{n}, Q}(u, v)=\max \left\{0, u+v-1, Q\left(x_{1}, y_{1}\right)-\left(x_{1}-u\right)^{+}-\left(y_{1}-v\right)^{+},\right. \\
Q\left(x_{2}, y_{2}\right)-\left(x_{2}-u\right)^{+}-\left(y_{2}-v\right)^{+}, \\
\left.Q\left(x_{2}-\frac{x_{2}-x_{1}}{n}, y_{1}\right)-\left(x_{2}-\frac{x_{2}-x_{1}}{n}-u\right)^{+}-\left(y_{1}-v\right)^{+}\right\}
\end{aligned}
$$

converges pointwise for all $(u, v) \in[0,1]^{2}$ to $B^{S, Q}(u, v)$. Finally, to prove that $B^{S, Q}$ is a copula, we need to verify the boundary conditions and the 2 -increasing property. Both elements are obviously satisfied when the sequence converges pointwise. In fact, if the pointwise limit of a sequence of copulas exists at each point of $[0,1]^{2}$ then the limit must be a copula (see the comment after Definition 3.3.4 of Nelsen (2006, p. 97)). The same limiting arguments can be used to show that $B^{S, Q}$ is a copula when $S$ is a nondecreasing compact set that contains a vertical part and a horizontal part that are both disconnected (so that both (2.3) and (2.6) are not satisfied). In this sense, the results of Tankov (2011) appear as a special case of ours. 


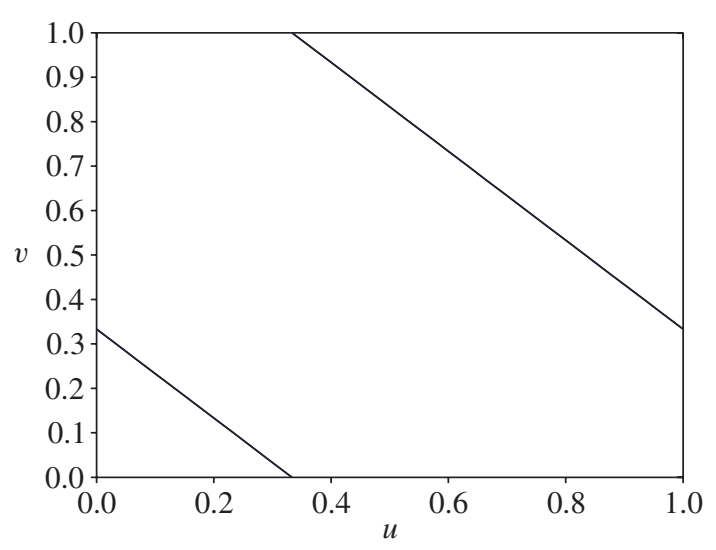

FIgURE 1: The support of the copula in Example 2.1.

Corollary 2.1. Let $Q$ be a copula, and let $S \subseteq[0,1]^{2}$ be a compact convex set satisfying there exist $\left(a_{0}, b_{0}\right) \in S$ and $\left(a_{1}, b_{1}\right) \in S$ for all $(u, v) \in S, a_{0} \leq u \leq a_{1}, b_{0} \leq v \leq b_{1}$.

Then $B^{S, Q}$ is a copula.

Proof. We prove that $\gamma_{1}$ and $\gamma_{2}$ are nondecreasing on $\left(a_{0}, a_{1}\right)$, and apply Theorem 2.1 (since property (2.3) is satisfied). Indeed, by the convexity of $S$, for any two points $\left(x_{1}, \gamma_{2}\left(x_{1}\right)\right)$ and $\left(x_{2}, \gamma_{2}\left(x_{2}\right)\right)$, we have $\left(\left(x_{1}+x_{2}\right) / 2,\left(\gamma_{2}\left(x_{1}\right)+\gamma_{2}\left(x_{2}\right)\right) / 2\right) \in S$. We can conclude that

$$
\frac{\gamma_{2}\left(x_{1}\right)+\gamma_{2}\left(x_{2}\right)}{2} \leq \max \left\{v \mid\left(\frac{x_{1}+x_{2}}{2}, v\right) \in S\right\}=\gamma_{2}\left(\frac{x_{1}+x_{2}}{2}\right)
$$

thus, $\gamma_{2}$ is concave. Similarly, $\gamma_{1}$ is convex. Finally, since $\gamma_{2}$ is concave, $R\left(x_{1}, x_{2}\right)=\left(\gamma_{2}\left(x_{2}\right)-\right.$ $\left.\gamma_{2}\left(x_{1}\right)\right) /\left(x_{2}-x_{1}\right)$ is nonincreasing in $x_{1}$ for $x_{2}$ fixed, and in $x_{2}$ for $x_{1}$ fixed. Therefore, $\gamma_{2}$ is nondecreasing on $\left[a_{0}, a_{1}\right]$ because of property (2.7). A similar reasoning shows that $\gamma_{1}$ is also nondecreasing.

Note that Corollary 2.1 is not valid when the compact set $S$ is simply convex and compact as shown by the following example.

Example 2.1. Let $S$ be the line connecting $\left(\frac{1}{3}, \frac{2}{3}\right)$ with $\left(\frac{2}{3}, \frac{1}{3}\right)$. Let $Q$ be the copula defined by the support in Figure 1, namely,

$$
Q(u, v)= \begin{cases}\max \left\{u+\min \left\{v, \frac{1}{3}\right\}-\frac{1}{3}, 0\right\}, & v \in\left[0, \frac{1}{3}\right] \\ \max \left\{u+v-1, \min \left\{v, \frac{1}{3}\right\}\right\}, & v \in\left[\frac{1}{3}, 1\right]\end{cases}
$$

It can be easily shown that $Q$ takes the constant value $\frac{1}{3}$ on $S$. Observe that

$$
B^{S, Q}\left(\frac{2}{3}, \frac{2}{3}\right)=\frac{1}{3}, \quad B^{S, Q}\left(\frac{1}{3}, \frac{2}{3}\right)=\frac{1}{3}, \quad B^{S, Q}\left(\frac{2}{3}, \frac{1}{3}\right)=\frac{1}{3}, \quad \text { and } \quad B^{S, Q}\left(\frac{1}{3}, \frac{1}{3}\right)=0 .
$$

Therefore, on the rectangle $\left[\frac{1}{3}, \frac{2}{3}\right]^{2}, B S, Q$ is not 2-increasing.

Theorems 2.1 and 2.2 show that there is a wide class of convex compact sets such that $B^{S, Q}$ given by (1.1) is a copula. Similar results can be obtained for $A^{S, Q}$. 
Theorem 2.3. Let $Q$ be a copula, and let $S \subseteq[0,1]^{2}$ be a compact set.

(i) If $\gamma_{1}$ and $\gamma_{2}$ are nonincreasing functions and $S$ satisfies (2.3), then $A^{S, Q}$ is a copula.

(ii) If $\gamma_{3}$ and $\gamma_{4}$ are nonincreasing functions and $S$ satisfies (2.6), then $A^{S, Q}$ is a copula.

Proof. (i) Similarly as in the proof of Theorem 1 of Tankov (2011) we note that

$$
A^{S, Q}(u, v)=\overline{B^{\bar{S}, \bar{Q}}}(u, v),
$$

where

$$
\bar{S}=\{(a, b) \mid(a, 1-b) \in S\} \quad \text { and } \quad \bar{Q}(u, v)=u-Q(u, 1-v) .
$$

The nonincreasing property of $\gamma_{1}$ and $\gamma_{2}$ implies that $\bar{\gamma}_{1}$ and $\bar{\gamma}_{2}$ (defined in an obvious way for $\bar{S})$ are nondecreasing. Since $\bar{Q}$ is a copula, the first part of the proof implies that $B^{\bar{S}}, \bar{Q}(u, v)$ is copula; hence, $A^{S, Q}(u, v)=\overline{B^{\bar{S}}, \bar{Q}}(u, v)$ is also a copula. The proof of (ii) is similar.

As an immediate result of Theorems 2.1 and 2.3, we have the following corollary.

Corollary 2.2. For any copula $Q$ and any rectangle $S=\left[u_{1}, u_{2}\right] \times\left[v_{1}, v_{2}\right]$ in the unit square, $A^{S, Q}$ and $B S, Q$ are both copulas.

Proof. For a rectangle $S, \gamma_{1}$ and $\gamma_{2}$ (as defined by (2.1) and (2.2)) are clearly nondecreasing and nonincreasing, and property (2.3) is obviously satisfied. Therefore, using Theorems 2.1 and $2.3, A^{S, Q}$ and $B^{S, Q}$ are both copulas.

\section{Application to optimal investment strategies}

Let $(\Omega, \mathcal{F}, \mathrm{P})$ be a probability space describing a financial market. Using a suitable equilibrium model or no-arbitrage arguments, financial theory shows that the price of a strategy with terminal payoff $X_{T}$ (paid at time $T>0$ ) can be written as

$$
c\left(X_{T}\right)=\mathrm{E}\left[\xi_{T} X_{T}\right],
$$

where $\xi_{T}$ is some given stochastic discount factor (also called the state-price process at $T$ ), and where it is tacitly assumed that expectation (3.1) exists. In fact, for $\omega \in \Omega, \xi_{T}(\omega)$ can be interpreted as the price of consuming one unit in state $\omega$ and 0 in all others. It is high in the worst states of the economy, that is, when the 'market' is at its lowest level.

For investors with increasing law-invariant preferences (i.e. when they only consider the distribution of terminal wealth and prefer more to less), it can be shown that optimal strategies are obtained by minimizing the price $\mathrm{E}\left[\xi_{T} X_{T}\right]$ such that $X_{T}$ has a given final distribution. Note that $f(x, y)=x y$ is 2-increasing; hence, minimizing $\mathrm{E}\left[\xi_{T} X_{T}\right]$ for fixed marginals is equivalent to minimizing the copula $C$ of $\left(\xi_{T}, X_{T}\right)$; see Meilijson and Nadas (1979), Rüschendorf (1983), and Tchen (1980). Hence, it becomes clear that optimal strategies are anti-monotonic with $\xi_{T}$. In other words, the copula $C$ corresponds to the Fréchet-Hoeffding lower bound; see also Bernard et al. (2010).

It thus also turns out that such optimal strategies exhibit their worst outcomes when $\xi_{T}$ is high corresponding to bearish financial markets. In practice, investors may want to impose constraints on the copula $C$, reflecting that they take into account the states where cash flows are received, or, more precisely, the dependence between the strategy and the financial market. The constraints can be described by a set $S$ and a copula $Q$. When $B^{S, Q}(u, v)$ is a copula, the optimal investment strategy can be constructed. This idea has recently been pursued in Bernard 

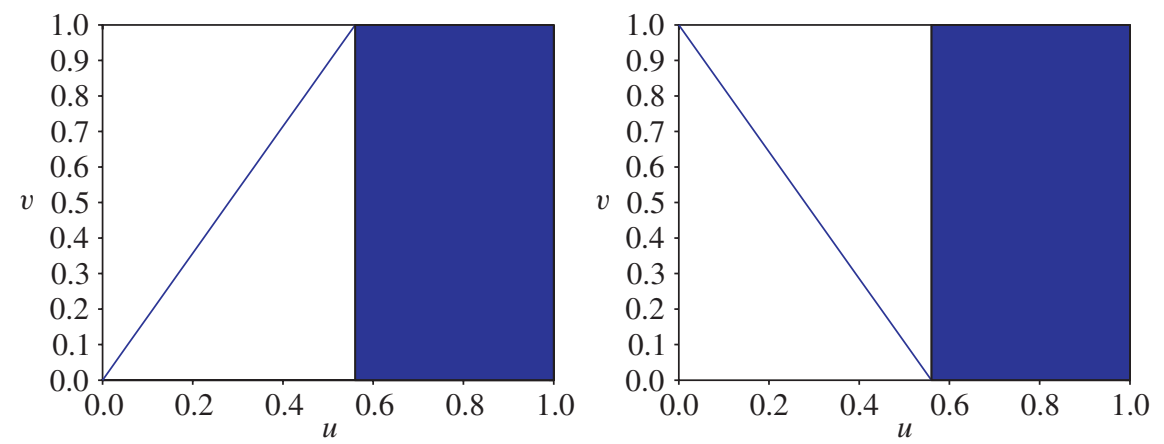

Figure 2: Supports for the copulas $A^{S, Q}($ left $)$ and $B^{S, Q}$ (right) of Example 3.1 with $u_{0}=0.56$. The supports are obtained by simulation using the facts that $\left(\partial A^{S, Q} / \partial u\right)^{-1}(y)=\left(u / u_{0}\right) \mathbf{1}_{\left\{u \leq u_{0}\right\}}+y \mathbf{1}_{\left\{u>u_{0}\right\}}$ and $(\partial B S, Q / \partial u)^{-1}(y)=\left(1-u / u_{0}\right) \mathbf{1}_{\left\{u \leq u_{0}\right\}}+y \mathbf{1}_{\left\{u>u_{0}\right\}}$.

and Vanduffel (2011) (for the case when $S$ is nondecreasing and compact). The theorems discussed in this note allow us to apply this idea to a much broader setting covering a variety of financially interesting situations. We now give an illustration.

Example 3.1. Let $Q(u, v)=u v$ be the independence copula. Let $S$ be given as

$$
S=\left\{(a, b) \in[0,1]^{2} \mid a \geq u_{0}, b \in[0,1]\right\} .
$$

Applying Corollary 2.2, we find after some calculations that the maximum copula $A^{S, Q}$ satisfying

$$
A^{S, Q}(u, v)=u v \quad \text { for all }(u, v) \in S
$$

is given by

$$
A^{S, Q}(u, v)=\min \left(u, u_{0} v\right) \mathbf{1}_{\left\{u \leq u_{0}\right\}}+u v \mathbf{1}_{\left\{u>u_{0}\right\}} .
$$

Similarly, we find that

$$
B^{S, Q}(u, v)=\max \left(0, u_{0}(v-1)+u\right) \mathbf{1}_{\left\{u \leq u_{0}\right\}}+u v \mathbf{1}_{\left\{u>u_{0}\right\}}
$$

is the minimum copula. The supports of $A^{S, Q}$ and $B^{S, Q}$ are represented graphically in Figure 2.

The minimum copula obtained in Example 3.1 allows us to construct a strategy that provides, at the lowest possible cost, the desired distribution which also exhibits independence with the market when the latter is low (high states for $\xi_{T}$ ). This is thus a very useful strategy for investors who seek diversification (i.e. some degree of protection) in times of a crisis.

\section{Acknowledgements}

The authors gratefully acknowledge the program 'Brains Back to Brussels' that funded an extended research visit of C. Bernard at Vrije Universiteit Brussel in Brussels during which this paper was initiated. S. Vanduffel acknowledges the financial support of the BNP Paribas Fortis Chair in Banking. C. Bernard also acknowledges support from the Natural Sciences and Engineering Research Council of Canada. X. Jiang was funded by an NSERC-USRA when the project was written. The authors would like to thank Roger Nelsen for stimulating discussions. They also thank an anonymous referee for very helpful and constructive comments, and in particular for a suggestion that allowed us to substantially relax the conditions of Theorem 1.1. 


\section{References}

Bernard, C. And Vanduffel, S. (2011). Optimal investment under probability constraints. In Proc. 2011 Actuarial and Financial Mathematics Conf., Universa Press, Belgium, pp. 3-14.

Bernard, C., Boyle, P. P. and Vanduffel S. (2011). Explicit representation of cost-efficient strategies. Working paper. Available at http://dx.doi.org/10.2139/ssrn.1561272.

MeiliJson, I. And Nadas, A. (1979). Convex majorization with an application to the length of critical paths. J. Appl. Prob. 16, 671-677.

Nelsen, R. (2006). An Introduction to Copulas, 2nd edn. Springer, New York.

RACHEV, S. T. AND RÜSCHENDORF, L. (1994). Solution of some transportation problems with relaxed or additional constraints. SIAM J. Control Optimization 32, 673-689.

RaChev, S. T. AND RÜSChendorf, L. (1998). Mass Transportation Problems, Vol. II. Springer, New York.

RüSCHENDORF, L. (1983). Solution of a statistical optimization problem by rearrangement methods. Metrika 30, 55-61.

TANKov, P. (2011). Improved Fréchet bounds and model-free pricing of multi-asset options. J. Appl. Prob. 48, 389-403.

Tchen, A. H. (1980). Inequalities for distributions with given margins. Ann. Appl. Prob. 8, 814-827. 